\title{
A Rare Case of Atrial Metastasis From a Rectal Adenocarcinoma
}

\author{
Srinivas S. Ayyala ${ }^{a}$, Dawnette M. Urcuyo ${ }^{a}$, Paul T. Kannarkatt ${ }^{\mathrm{a}}$, James E. Kovacs ${ }^{\mathrm{b}}$, \\ Nicole J. Terrigno a, c
}

\begin{abstract}
Colorectal cancers typically metastasize to the lymph nodes, liver or lungs. Metastasis to the heart is rare and although a few cases of cardiac metastases from colon cancer are described in the literature, cases of metastatic rectal cancer to the heart are far fewer. A 69-yearold woman with a history of rectal adenocarcinoma treated with neo-adjuvant chemotherapy and radiation, followed by resection and adjuvant chemotherapy, presented with increasing dyspnea on exertion and lower extremity edema 5 years after oncology follow-up. Echocardiography revealed a mass within the right atrium, which was biopsied and found to be consistent with metastatic rectal adenocarcinoma and a thrombus. The patient was deemed to be a poor surgical candidate given her co-morbidities and overall prognosis. Chemotherapy was offered and refused by the patient. The medical literature has a paucity of similar cases of rectal adenocarcinoma metastasizing to the right atrium. Further studies are needed to help guide standardized treatment options.
\end{abstract}

Keywords: Colorectal cancer; Rectal adenocarcinoma; Cardiac metastasis; Cardiac tumors

\section{Introduction}

Metastatic cancers affecting the heart are relatively rare but do cite back to the 1940s, showing up on autopsy reports approximately $10 \%$ of the time [1-3]. Cardiac metastases may be underestimated given that they are often clinically silent.

Although there is potential for any malignancy to metastasize to the heart, colorectal cancers typically metastasize to the lymph nodes, liver or lungs, usually by lymphatic or hematogenous spread [3-5]. Other unusual sites of metastases from colorectal cancers have been reported in the literature,

Manuscript submitted July 9, 2017, accepted July 4, 2017

aDepartment of Medicine, Cooper University Hospital, Camden, NJ, USA ${ }^{b}$ Department of Radiology, Cooper University Hospital, Camden, NJ, USA ${ }^{\mathrm{c} C}$ Corresponding Author: Nicole Terrigno, Cooper University Hospital-Cooper Medical School of Rowan University, 1 Cooper Plaza, Camden, NJ 08103, USA. Email: terrigno-nicole@cooperhealth.edu

doi: https://doi.org/10.14740/jocmr3123w such as to the spleen, skeletal muscle and thyroid gland [6-8]. Although a few cases of cardiac metastases from colon cancer are described in the literature [3, 4, 9-11], cases of metastatic rectal cancer to the heart are far fewer. In fact, we found only three such well-documented cases of cardiac metastasis from rectal adenocarcinoma in the literature [12-14].

Our report describes the case of a 69 -year-old female with a significant past medical history of rectal adenocarcinoma who presented with a 1-month history of worsening dyspnea on exertion after being lost to oncology follow-up for 5 years. Her symptoms were associated with fatigue and bilateral lower extremity swelling and she was ultimately found to have metastatic cancer to the right atrium.

\section{Case Report}

A 69-year-old Caucasian female with a 20-pack-year smoking history and a past history of rectal cancer (stage T3N1M0), treated with neo-adjuvant chemotherapy and radiation, followed by surgical resection with a low anterior resection, ileostomy formation and two subsequent cycles of adjuvant FOLFOX chemotherapy (further chemotherapy had to be aborted secondary to the development of severe neuropathy) presented for further evaluation of worsening dyspnea, fatigue and lower extremity edema 5 years after being lost to oncology follow-up. Her admission labs were significant for mild anemia, thrombocytopenia and acute kidney injury. A CT scan of the chest revealed multiple pulmonary nodules, ascites, a 2.5 $\mathrm{cm}$ retroperitoneal lymph node and a mass in the right atrium. A transesophageal echocardiogram revealed a large $4 \times 3.5 \mathrm{~cm}$ mass in the right atrium (Fig. 1). A cardiac MRI showed the same right atrial mass extending into the tricuspid valve leaflet and the inferior vena cava to the level of the intrahepatic inferior vena cava (Fig. 2). An MRI of the abdomen and pelvis confirmed a heterogeneous, hyper-intense filling defect in the inferior vena cava from the level of the renal veins extending into the right atrium (Figs. 3 and 4). The patient underwent a paracentesis for ascites, which yielded a transudative fluid without malignant cells (WBC 531, ascitic fluid albumin 1.2 $\mathrm{g} / \mathrm{dL}$, lactate dehydrogenase $269 \mathrm{U} / \mathrm{L}$ (normal $110-230 \mathrm{U} / \mathrm{L}$ ), and serum albumin $2.9 \mathrm{~g} / \mathrm{dL}$ (normal $3.8-5.3 \mathrm{~g} / \mathrm{dL}$ )). Serum carcinoembryonic antigen (CEA) and carbohydrate antigen 19-9 (CA 19-9) levels were elevated at $42.2 \mathrm{ng} / \mathrm{mL}$ (4 ng/mL in 2011) and $49 \mathrm{U} / \mathrm{mL}$, respectively. She underwent a cardiac catheterization with biopsy of the right atrial mass, which 


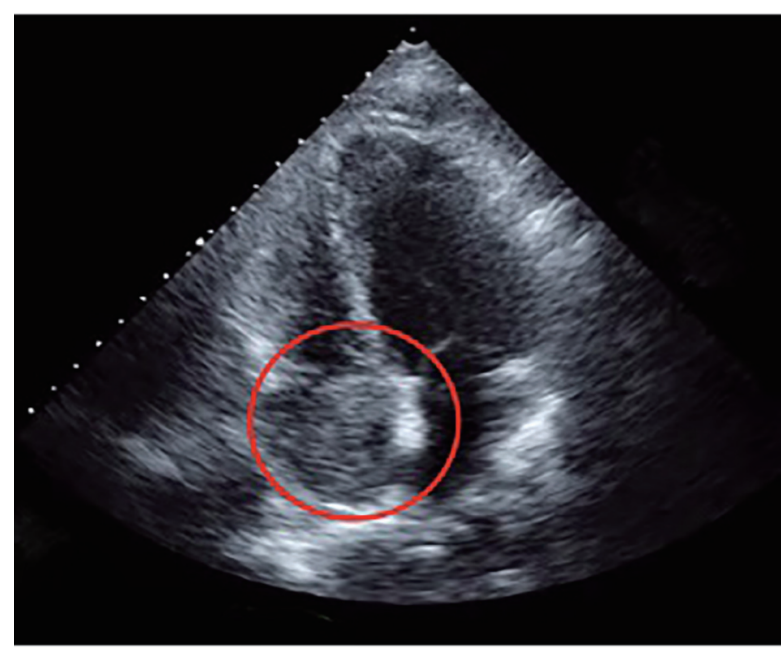

Figure 1. A transesophageal echocardiogram in a four-chamber view showing a right atrial mass, $4 \times 3.5 \mathrm{~cm}$.

revealed carcinoma cells positive for CDX2 and CK20 and negative for CK7 by immunohistochemistry, consistent with metastatic rectal adenocarcinoma. She was started on anticoagulation for the concomitant thrombus and was evaluated by the oncology team who recommended palliative chemotherapy. She declined chemotherapy and ultimately chose home hospice care instead.

\section{Discussion}

Although cardiac metastasis in any malignancy is rare, the incidence of metastatic disease to the heart may be underestimated because it is often clinically silent $[2,4,5]$. This phenomenon may occur for two reasons. First, the disease may be infiltrative and the tumor can compress and displace heart tissue rather than causing outright destruction, minimizing interference with cardiac contraction and conduction [2]. Secondly, as metastases can occur by hematogenous, transvenous or direct contiguous extension, they typically invade the peri-

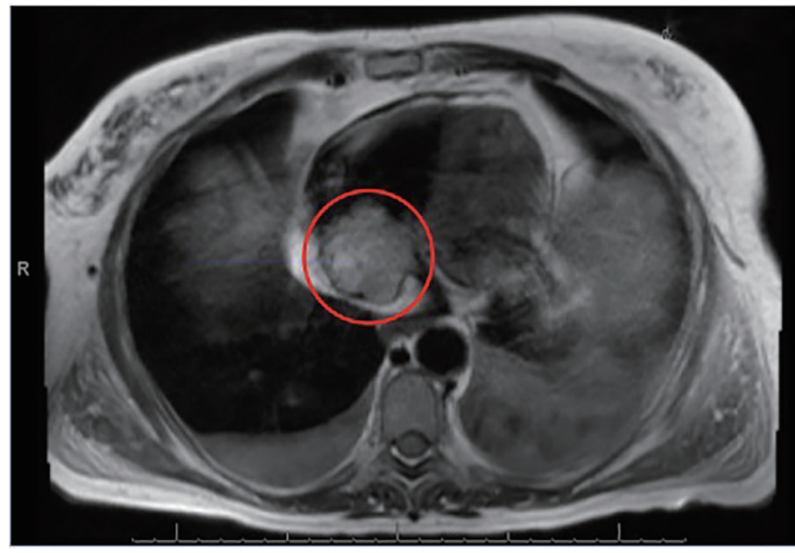

Figure 2. A T2-weighted MRI showing the right atrial, non-mobile and heterogeneously hyperintense mass, measuring $4.6 \times 4.2 \mathrm{~cm}$.

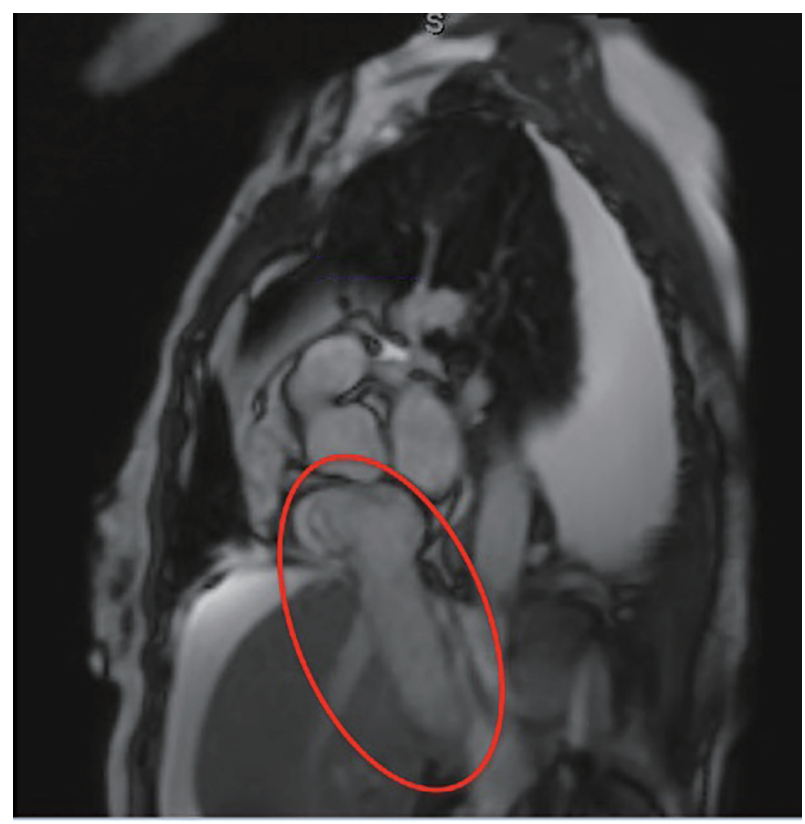

Figure 3. Sagittal oblique T2-weighted MRI of the chest showing a heterogeneously hyperintense filling defect in the inferior vena cava from the level of the renal veins into the right atrium. No hepatic mass was present.

cardium first, which may not initially produce symptoms, but can be associated with pericarditis or pericardial effusion in later stages $[1,4]$.

When patients do have symptoms of cardiac metastases,

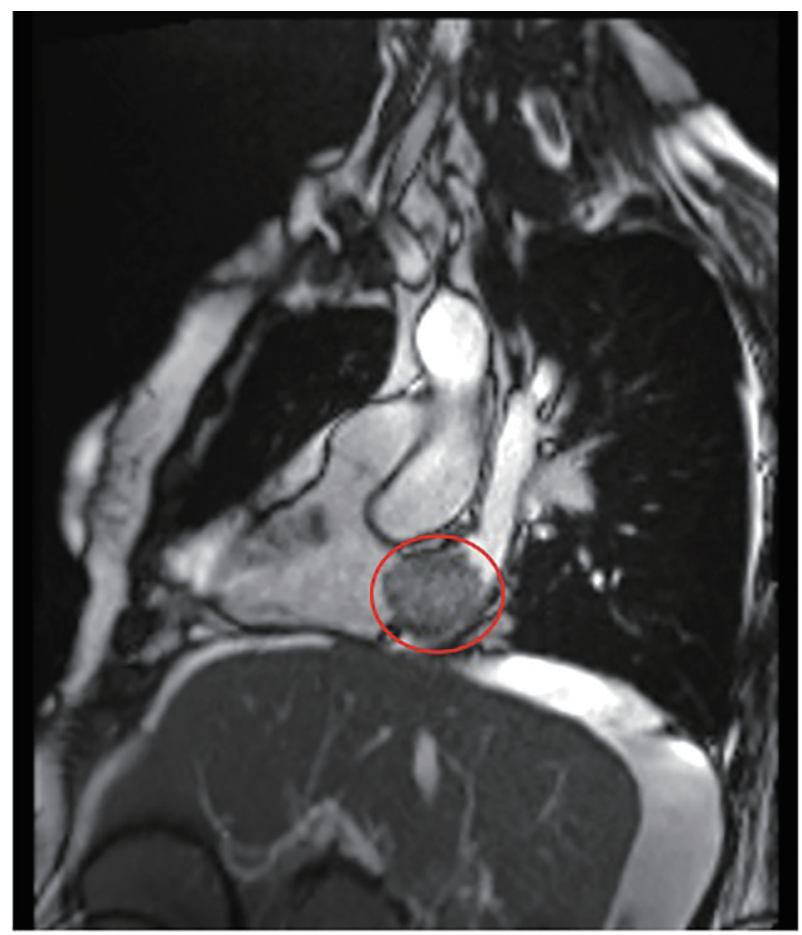

Figure 4. Sagittal oblique T2-weighted sequence through the right heart inflow and outflow tracts showing the right atrial mass in the right atrium. 
they are often non-specific, including symptoms of right-sided heart failure, dyspnea, arrhythmias, pulmonary thromboembolism or tumor thrombus extending to the inferior or superior vena cava, resulting in edema and obstruction $[4,10,11,15]$. As with our patient, tissue diagnosis is often necessary to determine the best course of management.

When evaluating cardiac masses, differential diagnoses should include primary endocardial tumors, such as atrial myxomas, vegetations or organized thrombi [4]. Cardiac masses are usually initially investigated using transesophageal echocardiography to confirm the presence of metastatic disease [4]. CT or MRI with contrast or newer modalities such as contrastinduced echocardiography are thought to provide a more panoramic view of the pericardium, myocardial walls and cardiac chambers, which may be useful in defining secondary cardiac tumors that are often infiltrative in nature. The contrast used in these scans may help distinguish a tumor from a thrombus $[4,5]$. Therefore, it seems reasonable that any patient with a history of malignancy and cardiopulmonary symptoms should undergo diagnostic testing for possible cardiac metastasis.

When definitively diagnosed, metastatic cardiac tumors carry a poor prognosis. If the metastasis is solitary, chemotherapy, radiation or resection may be helpful, but the metastatic burden and volume of disease are important considerations that often determine the treatment modality [15].

Surgical resection is often not feasible in the vast majority of patients with metastatic cardiac tumors due to disseminated metastatic disease. However, surgery may be indicated for palliation, when symptoms of hemodynamic compromise secondary to intra-cavitary tumor growth outweigh the risk of operative death, allowing more time for treatment with chemotherapy or radiation $[11,13]$. Determining surgical candidacy often consists of weighing the expected quality of life improvement or increase in life-expectancy against the perils of surgery, including the normal risks associated with cardiac bypass, the risk of further spread and seeding of the malignant cells and bleeding risk [12]. Chemotherapy, which was offered to our patient, is also an option, but is often palliative in nature. To date, there are no standardized approaches to treatment in patients with cardiac metastases from colorectal cancers and further studies are needed to delineate the best treatment course in this group of patients.

\section{Conclusion}

Although cardiac metastasis from malignancy is rare, the differential diagnosis should be considered in a patient with cardiopulmonary symptoms and a history of cancer. This case report sheds light on this diagnosis and brings awareness that to date, there are no standardized therapies in treating patients with cardiac metastases.

\section{References}

1. Hanfling SM. Metastatic cancer to the heart. Review of the literature and report of 127 cases. Circulation. 1960;22:474-483.

2. Goudie RB. Secondary tumours of the heart and pericardium. Br Heart J. 1955;17(2):183-188.

3. Choufani EB, Lazar HL, Hartshorn KL. Two unusual sites of colon cancer metastases and a rare thyroid lymphoma. Case 2. Chemotherapy-responsive right artial metastasis from colon carcinoma. J Clin Oncol. 2001;19(15):35743575.

4. Patel SA, Herfel BM, Nolan MA. Metastatic colon cancer involving the right atrium. Tex Heart Inst J. 2012;39(1):79-83.

5. Choi PW, Kim CN, Chang SH, Chang WI, Kim CY, Choi HM. Cardiac metastasis from colorectal cancer: a case report. World J Gastroenterol. 2009;15(21):2675-2678.

6. Bigot P, Goodman C, Hamy A, Teyssedou C, Arnaud JP. Isolated splenic metastasis from colorectal cancer: report of a case. J Gastrointest Surg. 2008;12(5):981-982.

7. Choi PW, Kim CN, Kim HS, Lee JM, Heo TG, Park JH, et al. Skeletal muscle metastasis from colorectal cancer: report of a case. Journal of the Korean Society of Coloproctology. 2008;24(6):492.

8. Phillips JS, Lishman S, Jani P. Colonic carcinoma metastasis to the thyroid: a case of skip metastasis. J Laryngol Otol. 2005;119(10):834-836.

9. Zipoli A, Bartoli P, Fradella G, Sansoni M, Brandinelli A, Mazza F, Ieri A. [Right atrial metastasis as an initial clinical manifestation of adenocarcinoma of the colon]. Ann Ital Med Int. 1994;9(3):150-152.

10. Teixeira T, Timoteo H, Marcao I. Cardiac Metastasis from Colon Carcinoma. Acta Medica Portugesa. 1997;10:331334.

11. Nishida H, Grooters RK, Coster D, Soltanzadeh H, Thieman KC. Metastatic right atrial tumor in colon cancer with superior vena cava syndrome and tricuspid obstruction. Heart Vessels. 1991;6(2):125-127.

12. Koizumi J, Agematsu K, Ohkado A, Shiikawa A, Uchida T. Solitary cardiac metastasis of rectal adenocarcinoma. Jpn J Thorac Cardiovasc Surg. 2003;51(7):330-332.

13. Parravicini R, Fahim NA, Cocconcelli F, Barchetti M, Nafeh M, Benassi A, Grisendi A, et al. Cardiac metastasis of rectal adenocarcinoma. Surgical treatment. Tex Heart Inst J. 1993;20(4):296-298.

14. Lord RV, Tie H, Tran D, Thorburn CW. Cardiac metastasis from a rectal adenocarcinoma. Clin Cardiol. 1999;22(11):749.

15. Chiles C, Woodard PK, Gutierrez FR, Link KM. Metastatic involvement of the heart and pericardium: CT and MR imaging. Radiographics. 2001;21(2):439-449. 